Noname manuscript No.

(will be inserted by the editor)

\title{
Exploring the Pion phenomenology within a fully covariant constituent quark model
}

\author{
Giovanni Salmè, Emanuele Pace and Giovanni \\ Romanelli
}

Received: date / Accepted: date

\begin{abstract}
An overview of the vector and tensor Generalized Parton Distributions for a charged pion is presented. Such observables, belonging to the set of quantities fundamental for a detailed investigation of the hadronic inner dynamics, have been evaluated within a fully covariant Constituent Quark Model, based on a proper Ansatz of the pion Bethe-Salpeter Amplitude and the Mandelstam formula for matrix elements of operators acting on relativistic composite systems. Given the very encouraging results already obtained for the vector distribution of a charged pion, the model has been extended to the tensor one, and some preliminary calculations will be illustrated.
\end{abstract}

Keywords Pion Generalized Parton Distributions · Covariant Constituent Quark Model

\section{Introduction}

The non perturbative regime of Quantum Chromodynamics (QCD), namely the present theory of the strong interaction, is still a paramount challenge. In particular, achieving a complete 3D description of hadrons is the topic of many and coherent efforts, both on experimental and theoretical sides. The perturbative regime, with its fundamental feature, the asymptotic freedom, has been experimentally investigated in great detail,

The present research activity is partially supported by the Italian MIUR grant PRIN 2008.

Giovanni Salmè

Istituto Nazionale di Fisica Nucleare, Sezione di Roma, P.le A. Moro 2, I-00185, Italy

Tel.: +39-6-49914872

Fax: +39-6-4454749

E. Pace

Phys. Dept., "Tor Vergata" University and INFN Sezione di Tor Vergata, Via della Ricerca Scientifica 1, 00133 Rome, Italy

G. Romanelli

Phys. Dept., "Tor Vergata" University, Via della Ricerca Scientifica 1, 00133 Rome, Italy 
and this has allowed to access the short-distance behavior of the hadronic wave functions. But, in order to shed light on confinement, the other peculiar feature of QCD (deeply related to its non linearity), one has to move towards the external part of the hadron wave function.

From the theoretical point of view, the Light-front (LF) framework, with variables defined by: $a^{ \pm}=a^{0} \pm a^{3}$ and $\mathbf{a}_{\perp} \equiv\left\{a_{x}, a_{y}\right\}$, is very suitable for describing relativistic, interacting systems, like hadrons. Among the several motivations for adopting the LF framework, beyond the well-known fact that the dynamics of the light-cone is naturally described in terms of LF variables, one has to mention that the vacuum is almost trivial within a LF field theory [1. Therefore, e.g. for the pion, one can construct the following meaningful Fock expansion (with some caveat for the zero-mode contributions [1])

$$
|\pi\rangle=|q \bar{q}\rangle+|q \bar{q} q \bar{q}\rangle+|q \bar{q} g\rangle \ldots
$$

where the valence component $|q \bar{q}\rangle$ and the non valence ones are fully considered. Within our approach, based on a covariant description of the pion state (see [2,3] and references quoted therein), one can explore also the contributions beyond the valence term.

In recent years, it has been recognized that a wealth of information on the partonic structure of hadrons is encoded in the Generalized Parton Distributions (GPD) (see, e.g., Ref. 4]), as well as in the Transverse-momentum Distributions (TMD) (see, e.g., Ref. [5). Those observables, that can be experimentally accessed through Deep Virtual Compton Scattering and Semi-inclusive Deep Inelastic Scattering, respectively, are expressed by quantities invariant for LF-boosts, and allow one to parametrize matrix elements (between hadronic states) of relevant quark-quark or gluon-gluon correlators. Aim of our covariant, phenomenological analysis is the evaluation of the leading-twist pion GPD's.

\section{Generalized Parton Distributions}

The generalized parton distributions are off-diagonal (respect to the hadron fourmomenta, i.e. $p_{f} \neq p_{i}$ ) matrix elements of quark-quark (or gluon-gluon) correlator projected onto the Dirac basis (see, e.g., Ref. [6] for a thorough investigation of the pion case), and naturally summarize a lot of information, scattered in many observables, like e.g. electromagnetic (em) form factors or parton-distribution functions. In particular, the pion, i.e. the simplest hadron to be considered, has two leading-twist quark GPD's: i) the vector, or no spin-flip, GPD, $H_{\pi}^{I}(x, \xi, t)$, and ii) the tensor, or spinflip, GPD, $E_{\pi, T}^{I}(x, \xi, t)(I=I S, I V$ labels isoscalar and isovector GPD's). In order to avoid Wilson-line contributions, one can choose the light-cone gauge 4] and gets

$$
\begin{aligned}
& 2\left(\begin{array}{c}
H_{\pi}^{I S}(x, \xi, t) \\
H_{\pi}^{I V}(x, \xi, t)
\end{array}\right)=\int \frac{d k^{-} d \mathbf{k}_{\perp}}{2} \\
& \times \int \frac{d z^{-} d z^{+} d \mathbf{z}_{\perp}}{2(2 \pi)^{4}} e^{i\left[\left(x P^{+} z^{-}+k^{-} z^{+}\right) / 2-\mathbf{z}_{\perp} \cdot \mathbf{k}_{\perp}\right]}\left\langle p^{\prime}\left|\bar{\psi}_{q}\left(-\frac{1}{2} z\right) \gamma^{+}\left(\begin{array}{c}
1 \\
\tau_{3}
\end{array}\right) \psi_{q}\left(\frac{1}{2} z\right)\right| p\right\rangle= \\
& =\left.\int \frac{d z^{-}}{4 \pi} e^{i\left(x P^{+} z^{-}\right) / 2}\left\langle p^{\prime}\left|\bar{\psi}_{q}\left(-\frac{1}{2} z\right) \gamma^{+}\left(\begin{array}{c}
1 \\
\tau_{3}
\end{array}\right) \psi_{q}\left(\frac{1}{2} z\right)\right| p\right\rangle\right|_{\tilde{z}=0}
\end{aligned}
$$


and

$$
\begin{aligned}
& \frac{P^{+} \Delta^{j}-P^{j} \Delta^{+}}{P^{+} m_{\pi}}\left(\begin{array}{c}
E_{\pi T}^{I S}(x, \xi, t) \\
E_{\pi T}^{I V}(x, \xi, t)
\end{array}\right)= \\
& =\left.\int \frac{d z^{-}}{4 \pi} e^{i\left(x P^{+} z^{-}\right) / 2}\left\langle p^{\prime}\left|\bar{\psi}_{q}\left(-\frac{1}{2} z\right) i \sigma^{+j}\left(\begin{array}{c}
1 \\
\tau_{3}
\end{array}\right) \psi_{q}\left(\frac{1}{2} z\right)\right| p\right\rangle\right|_{\tilde{z}=0}
\end{aligned}
$$

where $\tilde{z} \equiv\left\{z^{+}=z^{0}+z^{3}, \mathbf{z}_{\perp}\right\}, \psi_{q}(z)$ is the quark-field isodoublet and

$$
x=\frac{k^{+}}{P^{+}}, \quad \xi=-\frac{\Delta^{+}}{2 P^{+}}, \quad t=-\Delta^{2}, \quad \Delta=p^{\prime}-p \quad, \quad P=\frac{p^{\prime}+p}{2}
$$

with $\left\{k^{+}-\Delta^{+} / 2, \mathbf{k}_{\perp}-\Delta_{\perp} / 2\right\}$ the initial LF momentum of the active quark. Notice that the factor of two multiplying the vector GPD is chosen in order to obtain the em form factor from $\int d x H(x, \xi, t)$ (see below).

As anticipated above, there exist basic relations between the GPD's and other relevant observables. If we consider a charged pion, the vector GPD is related both to the parton distributions, $q(x)$, and to the em form as follows

$$
\begin{aligned}
& H_{\pi}^{u}(x, 0,0)=\theta(x) u(x)-\theta(-x) \bar{u}(-x) \\
& F_{\pi}(t)=\int_{-1}^{1} d x H_{\pi}^{I V}(x, \xi, t)=\int_{-1}^{1} d x H_{\pi}^{u}(x, \xi, t)
\end{aligned}
$$

where $H_{\pi}^{u}=H_{\pi}^{I S}+H_{\pi}^{I V}$. The relation between the non-spin flip GPD and the em form factor of a charged pion can be generalized, if one considers Mellin moments of both vector and tensor GPD's, obtaining the so-called Generalized Form Factors (GFF). In particular, for the $u$-quark vector and tensor GPD's, the corresponding Mellin moments are given by

$$
\begin{aligned}
& \int_{-1}^{1} d x x^{n} H_{\pi}^{u}(x, \xi, t)=\sum_{i=0}^{\ell}(2 \xi)^{i} A_{n+1,2 i}^{u}(t), \\
& \int_{-1}^{1} d x x^{n} E_{\pi, T}^{u}(x, \xi, t)=\sum_{i=0}^{\ell}(2 \xi)^{i} B_{n+1,2 i}^{u}(t)
\end{aligned}
$$

where $\ell=\{(n+1) / 2\}(\{a\}$ is the integer part of $a), A_{n+1,2 i}^{u}(t)$ is a vector GFF and $B_{n+1,2 i}^{u}(t)$ a tensor one. One has isoscalar (isovector) GFF's if $n$ is odd (even). It is worth noting that

$$
\int_{-1}^{1} d x H_{\pi}^{u}(x, \xi, t)=A_{1,0}^{u}(t)=F_{\pi}(t)
$$

and

$$
\int_{-1}^{1} d x E_{\pi, T}^{u}(x, \xi, t)=B_{1,0}^{u}(t)
$$

with $B_{1,0}^{u}(0) \neq 0$, the tensor charge for $n=1$, or the tensor anomalous magnetic moment (as it is called in Ref. [7]). 
In order to attach a physical interpretation to the GFF, one can generalize to a relativistic framework what is familiar for the em form factors (scalars and then boostinvariant observables) in a non relativistic approach. In this case, one interprets the 3D Fourier transforms of the em form factors of a hadron as the intrinsic (Galileaninvariant) em distributions in the coordinate space (e.g., for the pion, one has the charge distribution, while, for the nucleon, one has both charge and magnetic distributions). In the relativistic case, one has to consider Fourier transforms of GPD's, that are quantities depending upon variables invariant under LF boosts. Moreover, only the transverse part of $\Delta^{\mu}$ can be trivially conjugated to coordinate-space variables, while for $x$ and $\xi$ (proportional to $\Delta^{+}$) this is not possible. Therefore one can introduce bidimensional Fourier transforms with respect to $\boldsymbol{\Delta}_{\perp}$, for gaining some insight in the spatial distributions of the quarks, still keeping the description invariant for proper boosts (i.e. LF boosts). In particular, from Eq. (5) one can see that, putting $\xi=0$, only $A_{n+1,0}^{u}\left(\Delta^{2}\right)$ (cf also Eq. $\left.(5)\right)$ and $B_{n+1,0}^{u}\left(\Delta^{2}\right)$ survive. There exist an infinite set of frame where $\xi=0$, due to the LF-invariance of $\xi$, that are indicated as the Drell-Yan frames. In these frames, where $\Delta^{+}=0$ and $\Delta^{2}=-\Delta_{\perp}^{2}$, one can introduce the Fourier transforms of the previously defined GFF's as follows

$$
\tilde{A}_{n}\left(b_{\perp}\right)=\int \frac{d \boldsymbol{\Delta}_{\perp}}{(2 \pi)^{2}} e^{i \boldsymbol{\Delta}_{\perp} \cdot \mathbf{b}_{\perp}} A_{n, 0}\left(\Delta^{2}\right), \tilde{B}_{n}\left(b_{\perp}\right)=\int \frac{d \boldsymbol{\Delta}_{\perp}}{(2 \pi)^{2}} e^{i \boldsymbol{\Delta}_{\perp} \cdot \mathbf{b}_{\perp}} B_{n, 0}\left(\Delta^{2}\right)(8)
$$

where $b_{\perp}=\left|\mathbf{b}_{\perp}\right|$. They have a direct physical interpretation as quark densities in the impact-parameter space (IPS) 8, 9. In particular, $\tilde{A}_{n}\left(b_{\perp}\right)$ represents the probability density of finding an unpolarized quark in the pion at a certain distance $b_{\perp}$ from the transverse center of momentum. If one takes into account the quark polarization, then the probability density of finding in the Drell-Yan frame a charged parton of fixed transverse polarization, $\mathbf{s}_{\perp}$, and given $\mathbf{b}_{\perp}$, is

$$
\rho_{n}\left(\mathbf{b}_{\perp}, \mathbf{s}_{\perp}\right)=\frac{1}{2}\left[\tilde{A}_{n}\left(b_{\perp}\right)+\frac{s^{i} \epsilon^{i j} b^{j}}{b_{\perp}} \Gamma_{n}\left(b_{\perp}\right)\right]
$$

where

$$
\Gamma_{n}\left(b_{\perp}\right)=-\frac{1}{2 m_{\pi}} \frac{\partial \tilde{B}_{n}\left(b_{\perp}\right)}{\partial b_{\perp}}
$$

The IPS density $\rho_{n}$ can be obtained by calculating the proper matrix elements of the projector for a transversely-polarized quark $\left(s^{\mu} \equiv\left\{0,0, \mathbf{s}_{\perp}\right\}\right)$, namely

$$
\frac{1}{2} \gamma^{+}\left[1+\mathbf{s}_{\perp} \cdot \gamma_{\perp} \gamma_{5}\right]=\frac{1}{2}\left[\gamma^{+}-i \sum_{j} s^{j} \sigma^{+j} \gamma_{5}\right]=\frac{1}{2}\left[\gamma^{+}-\sum_{i j} s^{j} \sigma^{+i} \epsilon^{j i}\right]
$$

It should be pointed out that the distribution density of a quark with a given helicity (longitudinal polarization) is expressed by the first term in Eq. (9), since the pion is a pseudoscalar meson and the quark helicity operator (for a massless fermion: $\gamma_{5} / 2$ ) has a vanishing expectation value, due to parity invariance.

Equation (9) clearly indicates how to access the quark distribution in the impactparameter space through the study of the GPD's. Moreover, as a closing remark, one could exploit $E_{\pi T}$ to extract more elusive information on the quasi-particle nature of the constituent quarks, like their possible anomalous magnetic moments, once the vector current that governs the quark-photon coupling, is suitably improved (see below and 10] for a discussion in the lattice framework). 


\section{Transverse momentum distributions}

The transverse momentum distributions are diagonal (in the pion four-momentum) matrix elements of the quark-quark (or gluon-gluon) correlator with the proper Wilsonline contributions (see, e.g., Ref. [6]) and suitable Dirac structures. The TMD's depend upon $x$ and the quark transverse momentum, $\mathbf{k}_{\perp}$ (notice that $\Delta^{\mu}=0$ leads to $\xi=$ $t=0$ ), and since the integration over $\mathbf{k}_{\perp}$ is not performed, the Wilson-line effects must be carefully analyzed. At the leading-twist one has two TMD's, for the pion: the T-even $f_{1}\left(x,\left|\mathbf{k}_{\perp}\right|^{2}\right)$, that yields the probability distribution to find an unpolarized quark with LF momentum $\left\{x, \mathbf{k}_{\perp}\right\}$ in the pion, and the T-odd $h_{1}^{\perp}\left(x,\left|\mathbf{k}_{\perp}\right|^{2}, \eta\right)$, related to a transversely-polarized quark and called Boer-Mulders distribution [11]. The two TMD's allow one to parametrize the distribution of a quark with given LF momentum and transverse polarization, i.e. (see, e.g., Ref. [12])

$$
\rho\left(x, \mathbf{k}_{\perp}, \mathbf{s}_{\perp}\right)=\frac{1}{2}\left[f_{1}\left(x,\left|\mathbf{k}_{\perp}\right|^{2}\right)+\frac{s^{i} \epsilon^{i j} k_{\perp}^{j}}{m_{\pi}} h_{1}^{\perp}\left(x,\left|\mathbf{k}_{\perp}\right|^{2}, \eta\right)\right]
$$

where the dependence upon the variable $\eta$ in $h_{1}^{\perp}$ can be traced back to the Wilson-line effects. Moreover, it is worth reminding that $\mathbf{b}_{\perp}$ is not conjugated to $\mathbf{k}_{\perp}$, but to $\boldsymbol{\Delta}_{\perp}$.

By choosing the light-cone gauge and the advanced boundary condition for the gauge field, the effect of the Wilson lines (final state interaction effects) can be translated into complex phases affecting the initial state (see, e.g., Ref. [13]). At the lowest order, the unpolarized TMD $f_{1}^{I}\left(x,\left|\mathbf{k}_{\perp}\right|^{2}\right)$, acquaints the following form

$$
\begin{aligned}
& 2\left(\begin{array}{c}
f_{1}^{I S}\left(x,\left|\mathbf{k}_{\perp}\right|^{2}\right) \\
f_{1}^{I V}\left(x,\left|\mathbf{k}_{\perp}\right|^{2}\right)
\end{array}\right)= \\
& =\left.\int \frac{d z^{-} d \mathbf{z}_{\perp}}{2(2 \pi)^{3}} e^{i\left[x P^{+} z^{-} / 2-\mathbf{k}_{\perp} \cdot \mathbf{z}_{\perp}\right]}\left\langle p\left|\bar{\psi}_{q}\left(-\frac{1}{2} z\right) \gamma^{+}\left(\begin{array}{c}
1 \\
\tau_{3}
\end{array}\right) \psi_{q}\left(\frac{1}{2} z\right)\right| p\right\rangle\right|_{z^{+}=0},
\end{aligned}
$$

After integrating over $\mathbf{k}_{\perp}$, one gets the standard unpolarized parton distribution $q(x)$, viz

$$
q(x)=\int d \mathbf{k}_{\perp} f_{1}^{q}\left(x,\left|\mathbf{k}_{\perp}\right|^{2}\right)=H_{1}^{q}(x, 0,0)
$$

The T-odd TMD, $h_{1}^{\perp}\left(x,\left|\mathbf{k}_{\perp}\right|^{2}, \eta\right)$ vanishes at the lowest order in perturbation theory, since it becomes proportional to the matrix elements

$$
\left.\left\langle p\left|\bar{\psi}_{q}\left(-\frac{1}{2} z\right) i \sigma^{+j}\left(\begin{array}{c}
1 \\
\tau_{3}
\end{array}\right) \psi_{q}\left(\frac{1}{2} z\right)\right| p\right\rangle\right|_{z^{+}=0} \quad,
$$

that are equal to zero, due to the time-reversal invariance. In order to get a non vanishing Boer-Mulders distribution, one has to evaluate at least a first-order correction, involving Wilson lines (see, e.g., Refs. [14] and [6]). 


\section{The Covariant Constituent Quark Model}

Presently, extensive theoretical and experimental research programs are being pursued to gain information on both GPD's and TMD's of hadrons (particularly of the nucleon). Within such efforts, considering that the lattice calculations (in Euclidean space) cannot cover the whole kinematics that experiments can investigate, it seems worth considering the development of phenomenological models, that are able to include general features, like the covariance, and to properly describe both the experimental data and the lattice ones. In this way one could have an effective tool for the analysis of the results of the forthcoming experiments (e.g., the ones planned and approved at TJLAB), devoted to set-up the 3D tomography of hadrons.

The main ingredients in our covariant constituent quark model (CCQM) are represented by i) a model of the $4 \mathrm{D}$ quark-hadron vertex, namely the Bethe-Salpeter amplitude, and ii) the extension to the GPD's and TMD's of the 4D Mandelstam expression [15, originally introduced for calculating matrix elements of the em current operator for a relativistic interacting system. It is important to emphasize that our investigation, naturally goes beyond a purely valence description of the pion [2,3].

4.1 The Mandelstam Formula for the electromagnetic current

The Mandelstam formula allows one to express the matrix elements of the em current of a composite bound system, within a field theoretic approach [15]. It has been applied for evaluating the form factors of both pion [16] and nucleon [17. Furthermore, it has been exploited for calculating the vector GPD of the pion [2,3] and for a preliminary evaluation of the tensor GPD [18.

For instance, in the case of the em form factor of the pion, in the spacelike region, the Mandelstam formula within CCQM reads (see, e.g., Ref. [16])

$$
\begin{aligned}
& j^{\mu}=-\imath 2 e \frac{m^{2}}{f_{\pi}^{2}} N_{c} \int \frac{d^{4} k}{(2 \pi)^{4}} \bar{\Lambda}_{\pi}\left(k+q, P_{\pi}^{\prime}\right) \Lambda_{\pi}\left(k, P_{\pi}\right) \\
& \times \operatorname{Tr}\left[S\left(k-P_{\pi}\right) \gamma^{5} S(k+q) \Gamma^{\mu}(k, q) S(k) \gamma^{5}\right]
\end{aligned}
$$

where $f_{\pi}=92.4 \mathrm{MeV}$ is the pion decay constant, $N_{c}=3$ is the number of colors, $m=220 \mathrm{MeV}$ the CQ mass, $S(p)=\frac{1}{p-m+\imath \epsilon}$ the fermionic propagator, and $\Gamma^{\mu}(k, q)$ the quark-photon vertex, that simplifies to $\gamma^{\mu}$ in the spacelike region. In presence of a $\mathrm{CQ}$, one could add to the bare vector current a term proportional to an anomalous magnetic moment, namely a term like

$$
i \frac{\kappa_{q}}{2 m_{q}} \sigma^{\mu \nu} \Delta_{\nu}
$$

that should not be confused with the quark contribution to the nucleon anomalous magnetic moment. In the present approach we have disregarded such a possibility (cf [10. for an improved vector current within a lattice framework). In Eq. 16, it has been assumed a pion Bethe-Salpeter amplitude (BSA) with the following form

$$
\Psi(k-P, p)=-\frac{m}{f_{\pi}} S(k-\Delta / 2) \gamma^{5} \Lambda(k-P, p) S(k-P)
$$


where only the most important Dirac structure $\gamma_{5}$ has been retained (see Ref. [19] for a detailed discussion of terms beyond $\left.\gamma^{5}\right)$, and $\Lambda(k-P, p)$ is the momentum-dependent part.

The generalization to the case of GPD's, can be found in Ref. 2, 3. for the vector GPD, and in [18 20] for the tensor one. In particular, both an accurate description of the em form factor of the charged pion for $t \leq 0,2,3]$ and a nice behavior of the parton distribution evolved up to the experimental scale (see [20]) can be achieved by adopting the following analytic covariant Ansatz for the momentum-dependent part of the BSA

$$
\Lambda(k-P, p)=\frac{C}{\left[(k-\Delta / 2)^{2}-m_{R}^{2}+\imath \epsilon\right]\left[(P-k)^{2}-m_{R}^{2}+\imath \epsilon\right]}
$$

where the parameter $m_{R}=1200 \mathrm{MeV}$ is adjusted to fit $f_{\pi}$, while the constants $C$ is fixed through the form factor normalization, $F_{\pi}(t=0)=1$, that acts as a normalization condition for the BSA.

For the sake of concreteness, let us give both vector and tensor GPD's for the $u$ quark, viz

$$
\begin{aligned}
& 2 H^{u}(x, \xi, t)=-\imath \mathcal{R} \int \frac{d^{4} k}{(2 \pi)^{4}} \delta\left[P^{+} x-k^{+}\right] \Lambda\left(k-P, p^{\prime}\right) \Lambda(k-P, p) \\
& \times \operatorname{Tr}\left\{S(k-P) \gamma^{5} S(k+\Delta / 2) \gamma^{+} S(k-\Delta / 2) \gamma^{5}\right\}
\end{aligned}
$$

and

$$
\begin{aligned}
& \frac{P^{+} \Delta^{j}-P^{j} \Delta^{+}}{P^{+} m_{\pi}} E_{\pi T}^{u}(x, \xi, t)=i \mathcal{R} \int \frac{d^{4} k}{(2 \pi)^{4}} \delta\left[P^{+} x-k^{+}\right] \Lambda\left(k-P, p^{\prime}\right) \Lambda(k-P, p) \\
& \times \operatorname{Tr}\left[S(k-P) \gamma^{5} S(k+\Delta / 2) \gamma^{+} \gamma^{j} S(k-\Delta / 2) \gamma^{5}\right]
\end{aligned}
$$

where $\mathcal{R}=2 N_{c} m^{2} / f_{\pi}^{2}$ and $j=1,2$. The $\delta$ function imposes the correct support for the active quark, i.e. $|\xi| \leq x \leq 1$ (see 2, 3]), corresponding to the so-called DGLAP region 21. The diagrammatic representation of the vector GPD, with a LF-time ordering, is given in Fig. 1a and $1 \mathrm{~b}$.

$\Delta$

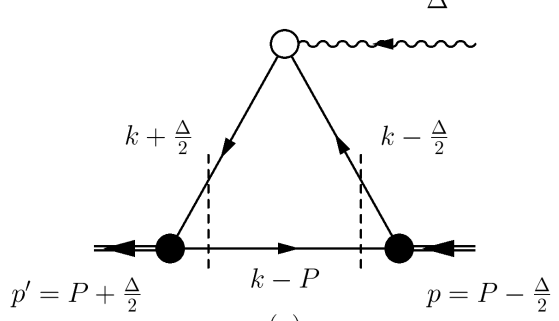

(a)

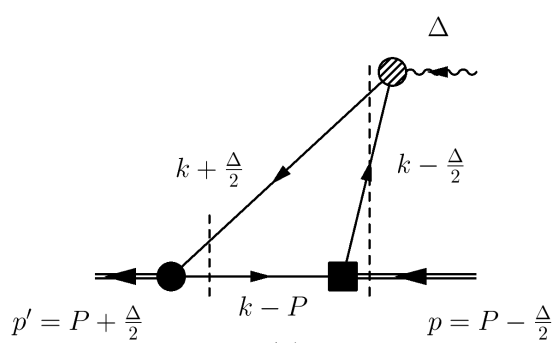

(b)

Fig. 1 LF time-ordered analysis of the pion GPD within the CCQM framework. Diagram (a): contribution of the active-quark in the valence region with $1 \geq x \geq|\xi|$. Diagram (b): contribution from the pair-production mechanism in the nonvalence region, $|\xi|>x>-|\xi|$. (After Ref. 203]). 

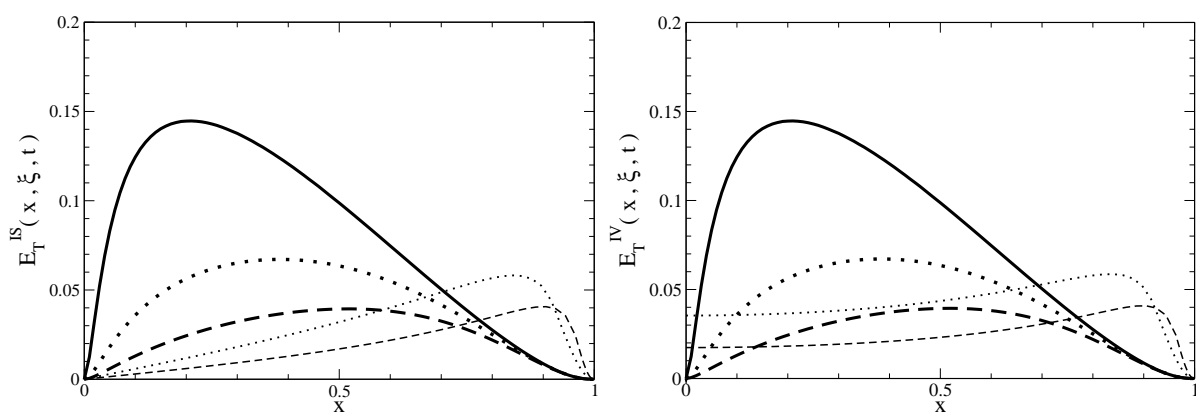

Fig. 2 Preliminary isoscalar and isovector tensor GPD's for a charged pion, within CCQM, for positive $x$. The behavior for negative values of $x$ can be obtained by recalling Eq. 21). Thick solid line: $\xi=0$ and $t=0$. Thick dotted line: $\xi=0$ and $t=-0.4 G e V^{2}$. Thick dashed line: $\xi=0$ and $t=-1 G e V^{2}$. Thin dotted line: $\xi=0.96$ and $t=-0.4 G e V^{2}$. Thin dashed line: $\xi=0.96$ and $t=-1 \mathrm{GeV}^{2}$.

In the CCQM investigation of GPD's, a Breit frame with $\Delta^{+}=-\Delta^{-} \geq 0$ is chosen in order to access the whole range of the variable $\xi$, i.e. $-1 \leq \xi \leq 1$. In this way both the valence and non valence region can be investigated, addressing the interesting topic of the smooth transition from the DGLAP (valence) regime to the ERBL (non valence) one 22. In Ref. 2 23, the vector GPD and the unpolarized TMD, resulting from the CCQM approach, are thoroughly analyzed. In particular, the CCQM calculation was able to carefully reproduce the experimental data of the em form factor of the charged pion over the whole spacelike range of $t$, presently explored. As to the GFF's obtained from the vector GPD (cf Eq. (5)), only lattice data exist and solely for $A_{2,0}(t)$ and $A_{2,2}(t)$ 23. Also for those GFF's, the comparison was quite encouraging.

In Fig. 2, our preliminary results for the tensor GPD of a charged pion are shown for some values of the variable $\xi$ and $t$, but for $0 \leq x \leq 1$. The GPD for negative values of $x$ can be obtained by exploiting the symmetry property of $E_{\pi T}^{I S, I V}(x, \xi, t)$ (see, e.g. Ref. [4) under the transformation $x \rightarrow-x$. The symmetry property follows from both the charge-conjugation $\left(p \rightarrow-p\right.$ and $\left.p^{\prime} \rightarrow-p^{\prime}\right)$ and the isospin invariance and leads to

$$
E_{\pi T}^{I S}(x, \xi, t)=-E_{\pi T}^{I S}(-x, \xi, t), \quad E_{\pi T}^{I V}(x, \xi, t)=E_{\pi T}^{I V}(-x, \xi, t)
$$

It is important to remind that for $\xi \rightarrow 0$ the valence component is dominant (DGLAP regime) while for $\xi \rightarrow 1$ the non valence term is acting (ERBL regime). In view of that, it is expected a peak around $x \sim 1$ for $\xi \rightarrow 1$, as discussed in Ref. 2, 3. Finally, from Eq. 21), one can understand the vanishing value of $E_{\pi T}^{I S}(0, \xi, t)$. In Ref. [18, our preliminary CCQM results have been compared with the calculations obtained within a LF Hamiltonian Dynamics framework, where only the valence component have been retained [3], obtaining a nice agreement in the relevant kinematical regions.

From the tensor GPD, one can calculate the corresponding GFF, see Eq. (5). We have started (see Ref. [18] for a preliminary presentation) the investigation by evaluating $B_{1,0}(t)$ and $B_{2,0}(t)$, and comparing our results with the lattice data 10,23 , as well as with other models like the chiral quark model of Ref. 24 and the instanton model of Ref. 25]. The complete analysis will be presented elsewhere 20. For a better comparison between our calculations of GFF's and the lattice results, we have also 


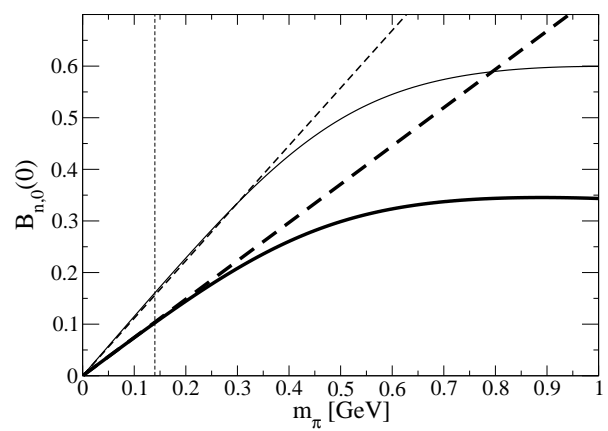

Fig. 3 Preliminary $B_{n, 0}(0)$ vs $m_{\pi}$, for $n=1,2$. Thin solid line: CCQM $B_{1,0}\left(0, m_{\pi}\right)$. Thin dashed line: $B_{1,0}^{\chi}\left(0, m_{\pi}\right)=1.13 m_{\pi}$. Thick solid line: CCQM $B_{2,0}\left(0, m_{\pi}\right)$. Thick dashed line: $B_{2,0}^{\chi}\left(0, m_{\pi}\right)=0.74 m_{\pi}$. As a guide, the physical pion mass is indicated by a vertical dashed line.

evaluated $B_{1,0}(t)$ and $B_{2,0}(t)$ both at the physical value of the pion mass, $m_{\pi}=140$ $\mathrm{MeV}$, and at the actual lattice value, $m_{\pi}^{\text {lat }}=600 \mathrm{MeV}$, tuning the CQ mass according to the Chiral Perturbation Theory (see, e.g. Ref. [26]), namely

$$
m_{q}=m_{q}^{\text {Phys }}+\left[\frac{m_{\pi}^{2}}{\left(m_{\pi}^{\text {Phys }}\right)^{2}}-1\right] m_{c}^{\text {Phys }}
$$

where $m_{q}^{\text {Phys }}$ and $m_{c}^{\text {Phys }}$ are the constituent quark mass and the current quark mass at the physical pion mass, respectively. In our approach the values $m_{q}^{\text {Phys }}=220 \mathrm{MeV}$ and $m_{c}^{\text {Phys }}=4 \pm 1 \mathrm{MeV}$ have been taken. Notice that lattice data were extrapolated to the physical pion mass by using a proper chiral formula [12,23. In particular, we can anticipate that the behavior of $B_{1,0}(t) / B_{1,0}(0)$ fully agrees with both i) the lattice data 12 and ii) the calculations from $\chi \mathrm{QM}$ [24] and instanton model [25], while for $B_{2,0}(t) / B_{2,0}(0)$ the comparison improves as the physical pion mass is approached. It is worth noting that the GFF's has been rescaled by their own value at $t=0$, in order to get rid off the effect of the QCD evolution on the tensor GPD (cf [24] and [25]). Furthermore, since the tensor charges of the pion are attracting an increasing interest (see also Ref. 27, where $B_{1,0}(0)$ is taken as a check for the lattice evaluation of other observables), it is important to carefully investigate $B_{n, 0}(0)$, for $n=1,2$, as a function of a running $m_{\pi}$, for getting information on their chiral limit (i.e. the relevant limit for lattice calculations). In Fig. 3, the preliminary results of CCQM for both $B_{1,0}\left(0, m_{\pi}\right)$ and $B_{2,0}\left(0, m_{\pi}\right)$ are shown; it is understood that in our analytic model, $B_{n, 0}\left(0, m_{\pi}\right)$ is evaluated by changing the values of $m_{\pi}$ and $m_{q}$, according to Eq. 22. It is worth noting that the linear behavior as a function of $m_{\pi}$, starts for $B_{2,0}\left(0, m_{\pi}\right)$ earlier than for $B_{1,0}\left(0, m_{\pi}\right)$, and in particular, for $m_{\pi} \leq 300 \mathrm{MeV}$ one has $B_{1,0}\left(0, m_{\pi}\right) \sim 1.13 m_{\pi}$ and $B_{2,0}\left(0, m_{\pi}\right) \sim 0.74 m_{\pi}$.

To accomplish a meaningful comparison with the lattice data, one has to evolve the CCQM results up to the scale $\mu=2 \mathrm{GeV}$, the scale of the lattice data. According to [24], the evolution should affect $B_{2,0}(0)$ more than $B_{1,0}(0)$ (almost a factor of two for $\mu_{0}=320 \mathrm{MeV}$ ). Though the complete investigation will be reported elsewhere [20, we can anticipate here that for $n=1$, the CCQM tensor charge is lower than the 
Table 1 Mean shifts along the direction perpendicular to the u-quark transverse polarization, $\mathbf{s}_{\perp} \equiv\{1,0\}$, for $n=1,2$.

\begin{tabular}{|c|c|c|}
\hline & CCQM $-m_{\pi}=140 \mathrm{MeV}$ & Lattice [12] \\
\hline \hline$\left\langle b_{y}\right\rangle_{1}$ & $0.113 \mathrm{fm}$ & $0.151 \pm 0.024 \mathrm{fm}$ \\
\hline$\left\langle b_{y}\right\rangle_{2}$ & $0.092 \mathrm{fm}$ & $0.106 \pm 0.028 \mathrm{fm}$ \\
\hline
\end{tabular}

lattice one presented in in Ref. 12, that in turn is bigger than the recent lattice data of Ref. 27. Furthermore, the comparison with the $\chi \mathrm{QM}$ calculation 24, in the limit of $m_{\pi} \rightarrow 0$, seems reasonable, since $B_{1,0}^{\chi Q M}(0) \sim 1.33 m_{\pi}$ and $B_{2,0}^{\chi Q M}(0) \sim 0.47 m_{\pi}$ at the quark-model scale $\mu_{0}=320 \mathrm{MeV}$.

The calculation of the GFF's, allows one to investigate the probability density $\rho_{n}\left(\mathbf{b}_{\perp}, \mathbf{s}_{\perp}\right)$ for a transversely-polarized u-quark (cf Eq. (9p) so that one can address the IPS structure of the pion. Table I shows preliminary results (still without evolution effects) for the average transverse shifts when the quark is polarized along the $x$-axis, i.e. $\mathbf{s}_{\perp} \equiv\{1,0\}$. The shift for a given $n$ is

$$
\left\langle b_{y}\right\rangle_{n}=\frac{\int d \mathbf{b}_{\perp} b_{y} \rho_{n}\left(\mathbf{b}_{\perp}, \mathbf{s}_{\perp}\right)}{\int d \mathbf{b}_{\perp} \rho_{n}\left(\mathbf{b}_{\perp}, \mathbf{s}_{\perp}\right)}=\frac{1}{2 m_{\pi}} \frac{B_{n, 0}(t=0)}{A_{n, 0}(t=0)}
$$

The values shown in Table I clearly demonstrate the dipole-like distortion of the transverse density in a direction perpendicular to the quark polarization, pointing to a non trivial correlation between the orbital angular momenta and the spin of the constituents inside a pseudoscalar hadron (see, e.g., Ref. 9 and references quoted therein).

\section{Conclusions and Perspectives}

A simple, fully covariant constituent quark model has been exploited for investigating the phenomenology of the leading-order Generalized Parton Distributions of the pion. The model has been already applied to the vector GPD [2,3, and in the present work some preliminary results for the tensor GPD have been illustrated. The main ingredients of the approach are i) an Ansatz for the Bethe-Salpeter amplitude and ii) the generalization of the Mandelstam formula, applied in the seminal work of Ref. [15] to matrix elements of the em current operator between states of a relativistic composite system.

The CCQM tensor GPD, $E_{\pi T}(x, \xi, t)$, favorably compares with the one obtained within the Light-front Hamiltonian dynamics (see 3,18]) and this represents an important test of the CCQM, as well as the analysis of the tensor charges by varying the pion mass (see Fig. 3), relevant for the chiral extrapolation of the lattice results. Finally the first evaluation of the transverse shifts of the probability distribution of a transversely-polarized quark has been given in Table I, indicating an encouraging comparison with the lattice data.

In order to construct a more and more reliable phenomenological tool, the CCQM study of the pion GPD will be improved by including the evolution with the energy scale, and by developing new Ansatzes of the Bethe-Salpeter amplitude, that allow one to increase the dynamical content, for instance within the Nakanishi perturbation integral representation of the quark-hadron vertex (see, e.g., Ref. 28. 


\section{References}

1. S. Brodsky, H-C Pauli, S. Pinsky, Quantum Chromodynamics and Other Field Theories on the Light Cone, Phys. Rep. 301, 299 (1998).

2. T. Frederico, E. Pace, B. Pasquini, and G. Salmè, Pion generalized parton distributions with covariant and light-front constituent quark models, Phys. Rev. D 80, 054021 (2009)

3. T. Frederico, E. Pace, B. Pasquini, and G. Salmè, Generalized parton distributions of the pion in a covariant Bethe-Salpeter model and light-front models, Nucl. Phys. B (Proc. Suppl.) 199, 264 (2010).

4. M. Diehl, Generalized parton distributions, Phys. Rep. 388, 41 (2003).

5. V. Barone, A. Drago, P. G. Ratcliffe, Transverse polarisation of quarks in hadrons, Phys. Rep. 359, 1 (2002).

6. S. Meißner, A. Metz, M. Schlegel, K. Goeke, Generalized parton correlation functions for a spin-0 hadron, JHEP 0808, 038 (2008).

7. Ph. Hägler, Hadron structure from lattice quantum chromodynamics, Phys. Rep. 490, 49 (2010).

8. M. Burkardt, Impact parameter dependent parton distributions and transverse single spin asymmetries, Phys. Rev. D 66, 114005 (2002).

9. M. Burkardt and B. Hannafious, Are all Boer-Mulders functions alike?, Phys. Lett. B 658, 130 (2008).

10. D. Brömmel et al., The pion form factor from lattice QCD with two dynamical flavours, Eur. Phys. J.C 51, 335(2007).

11. D. Boer and P. J. Mulders, Phys. Rev. D 57, 5780 (1998).

12. D. Brommel et al., Transverse spin structure of hadrons from lattice QCD, Pr. P. N. Phys. 61, 73 (2008); Spin Structure of the pion, Phys. Rev. Lett. 101, 122001 (2008).

13. A.V. Belitsky, X. Ji and F. Yuan, Final state interactions and gauge invariant parton distribution, Nucl. Phys. B 656, 165 (2003).

14. Zhun Lü and Bo-Qiang Ma, Nonzero transversity distribution of the pion in a quarkspectator-antiquark model, Phys. Rev. D 70, 094044 (2004).

15. S. Mandelstam, Dynamical variables in the Bethe-Salpeter formalism, Proc. Royal Soc. A 233, 248 (1956).

16. J.P.B.C. de Melo, T. Frederico, E. Pace and G. Salmè, Spacelike and timelike pion electromagnetic form-factor and Fock state components within the light-front dynamics, Phys. Rev. D 73, 074013 (2006); Electromagnetic form-factor of the pion in the space and time - like regions within the Front-Form dynamics, Phys. Lett. B 581, 75 (2004); Pair term in the electromagnetic current within the Front-Form dynamics: spin-0 case, Nucl. Phys. A 707, 399 (2002).

17. J.P.B.C. de Melo, T. Frederico, E. Pace, G. Salmè and S. Pisano, Timelike and spacelike nucleon electromagnetic form factors beyond relativistic constituent quark models, Phys. Lett. B 671,153 (2009).

18. E. Pace, G. Romanelli and G. Salmè, Pion Tensor Generalized Parton Distributions in a Covariant Constituent Quark Model, Few-Body Sys. in press, DOI 10.1007/s00601-0110276-z.

19. P. Maris and C. D. Roberts, $\pi$ and K-meson Bethe-Salpeter amplitudes, Phys. Rev. C 56, 3369 (1997).

20. E. Pace, G. Romanelli, G. Salme', to be published.

21. V. N. Gribov, L. N. Lipatov, Deep inelastic e-p scattering in perturbation theory, Sov. J. Nucl. Phys. 15, 438 (1972) [Yad. Fiz. 15, 781 (1972)]; e+ e- pair annihilation and deep inelastic e p scattering in perturbation theory, 15, 675 (1972); G. Altarelli, G. Parisi, Asymptotic freedom in parton language, Nucl. Phys. B 126, 298 (1977); Yu. L. Dokshitzer, Calculation of the Structure Functions for Deep Inelastic Scattering and e+ e- Annihilation by Perturbation Theory in Quantum Chromodynamics, Sov. Phys. JETP 46, 641 (1977).

22. A. V. Efremov, A. V. Radyushkin, Asymptotic behavior of the pion form factor in quantum chromodynamics, Phys. Lett. B 94, 245 (1980); G. P. Lepage, S. J. Brodsky, Exclusive processes in perturbative quantum chromodynamics, Phys. Rev. D 22, 2157 (1980).

23. D. Brömmel, Pion structure from the Lattice, Report No. DESY-THESYS-2007-023, 2007.

24. W. Broniowsky, Transversity form factors of the pion in chiral quark models, Phys. Rev. D 82, 094001 (2010).

25. S. Nam and H. Kim, Spin structure of the pion from the instanton vacuum, Phys. Lett. B 700, 305 (2011) 
26. I.C. Cloet, D. B. Leinweber and A. W. Thomas, Simple quark model with chiral phenomenology, Phys. Rev. C 65, 062201 (2002).

27. I. Baum, V. Lubicz, G. Martinelli, L. Orifici, and S. Simula, Matrix elements of the electromagnetic operator between kaon and pion states, Phys. Rev. D 84, 074503 (2011).

28. T. Frederico, G. Salmè and M. Viviani, Two-body scattering states in Minkowski space and the Nakanishi integral representation onto the null plane, Phys. Rev. D 85, 036009 (2012) and $\backslash$ protect \vrule width0pt \protect \href $\{$ http://arxiv.org/abs/1112.5568\}\{arXiv:1112.5568\}. 